\title{
Production and characterization of chitosomes for application as delivery system of bioactive compounds
}

\author{
Maria Fernanda Boldim Vaggione (IC), Mariano Michelon (PG), Rosiane Lopes da Cunha (PQ)
}

\begin{abstract}
Chitosomes are structures formed by electrostatic complexation of chitosan with phospholipid bilayers of liposome that aim to improve efficiency of liposomes in terms of stability and mucoadhesive properties as a delivery system of bioactive compounds. This study focused on analysis of operational parameters, particles characterization and study of chitosan-phospholipids interactions in order to obtain positively charged particle with nanometric size and low polidispersity index chitosomes.
\end{abstract}

Key words: phospholipid, chitosan, stability.

\section{Introduction}

Chitosomes are structures composed by phospholipids bilayers (liposomes), electrostatically complexed to chitosan, a biocompatible polysaccharide positively charged (which allows interaction with biological materials, which are mostly negatively charged). Chitosomes have potential utilization as a delivery system of bioactive compounds for food and pharmaceutical industry ${ }^{1}$. In spite of the advantages of conventional liposomes in vehiculation and delivery of bioactive compounds, they show limitations for an effective application, due to its low stability in gastrointestinal conditions. Alternatively, chitosomes show potential regarding stability, mucoadhesive properties and encapsulation efficiency ${ }^{2}$. Therefore, this work aimed the production of conventional liposomes and its electrostatic complexation with chitosan for future application as a more effective delivery system of bioactive compounds.

\section{Results and Discussion}

Liposomes were formerly produced from fat-free soybean lecithin by ethanol injection method, and complexation was performed using low molecular weight chitosan solutions $(0.05 \% \mathrm{w} / \mathrm{w})$ at $\mathrm{pH} 3.5$, at different chitosan:lecithin mass ratios. Phospholipid concentration in organic phase (100, 125 and $150 \mathrm{mM}$ ), and processing temperatures $\left(25\right.$ to $55^{\circ} \mathrm{C}$ ) were evaluated for production of liposomes. Chitosan concentration (0.0047 a $0.128 \% \mathrm{w} / \mathrm{w})$ and the effect of chitosan purification were studied for liposomes coating. Both systems were characterized by $\zeta$-potential, hydrodynamic diameter, polydispersity index (Pdl), Fourier Transform Infrared Spectrometry (FTIR) and Transmission Electron Microscopy (TEM). Liposomes were negatively charged ( $\zeta$-potential of -50 to $-60 \mathrm{mV}$ ) and showed unimodal size distribution, with mean diameter of 120 to $200 \mathrm{~nm}$ and Pdl values lower than 0.3. Lipossomes size tended to increase with lipids concentration whereas higher process temperature lead to smaller lipossomes mean diameter and greater Pdl. The condition selected for liposome coating by chitosan was of $150 \mathrm{mM}, 25^{\circ} \mathrm{C}$. Chitosan purification revealed to be promising for obtaining chitosomes of lower size and reduced Pdl. For chitosan:lecithin mass ratios of 0.023 to 0.0285 (\%w/w), chitosomes showed size range of 500 to $2000 \mathrm{~nm}$, approximately 0,18 Pdl and $\zeta$-potential of $+42 \mathrm{mV}$. TEM and FTIR analysis suggested interaction between liposome and chitosan.

\section{Conclusions}

Results showed that operational parameters for liposome production influenced its physicochemical characteristics, having been selected an optimal condition for further coating by chitosan. Different chitosan:lecithin mass ratios were studied for chitosomes production, and purification of chitosan was fundamental for obtaining particles of reduced size and low Pdl. Interaction between lecithin and chitosan was also evaluated for better understanding chitosomes organization. Production and characterization of chitosomes aimed the study of a more stable delivery system, alternative to conventional liposomes for delivery of bioactive compounds.

\section{Acknowledgement}

Acknowledgements to FAPESP (Fundação de Amparo à Pesquisa do Estado de São Paulo) for financial support.

\footnotetext{
Henriksen, I,; Vagen, SR.; Sande, S. A.; Smistad, G.; Karlsen, J. Interactions between liposomes and chitosan II. Effect of selected parameters on aggregation and leakage. International Journal of Pharmaceutics, v.146, p.103-204, 1997.

${ }^{2}$ Oku, N.; Namba, Y. Long-circulating liposomes. Critical Reviews in Therapeutic Drug Carrier Systems, v.11, p.231-270, 1994
} 